\title{
Bispectrum speckle interferometry of the Red Rectangle: Diffraction-limited near-infrared images reconstructed from Keck telescope speckle data
}

\author{
P. G. Tuthill ${ }^{1}$, A. B. Men'shchikov ${ }^{2}$, D. Schertl ${ }^{2}$, J. D. Monnier ${ }^{3}$, W. C. Danchi ${ }^{4}$, and G. Weigelt ${ }^{2}$ \\ 1 Astronomy Department, School of Physics, University of Sydney, NSW 2006, Australia \\ 2 Max-Planck-Institut für Radioastronomie, Auf dem Hügel 69, 53121 Bonn, Germany \\ e-mail: sasha@mpifr-bonn.mpg.de; ds@mpifr-bonn.mpg.de; weigelt@mpifr-bonn.mpg.de \\ 3 Smithsonian Astrophysical Observatory MS 42, 60 Garden Street, Cambridge, MA 02138, USA \\ e-mail: jmonnier@cfa.harvard.edu \\ 4 NASA Goddard Space Flight Center, Infrared Astrophysics, Code 685, Greenbelt, MD 20771, USA \\ e-mail: wcd@iri1.gsfc.nasa.gov
}

Received 19 December 2001 / Accepted 26 February 2002

\begin{abstract}
We present new near-infrared (2.1-3.3 $\mu \mathrm{m})$ images of the Red Rectangle with unprecedented diffractionlimited angular resolutions of 46-68 mas; 4 times higher than that of the Hubble space telescope and almost a factor of two improvement over the previous $6 \mathrm{~m}$ SAO telecope speckle images presented by Men'shchikov et al. (1998). The new images, which were reconstructed from Keck telescope speckle data using the bispectrum speckle interferometry method, clearly show two bright lobes above and below the optically thick dark lane obscuring the central binary. X-shaped spikes, thought to trace the surface of a biconical flow, change the intensity distribution of the bright lobes, making them appear broadened or with an east-west double-peak in images with the highest resolution. The striking biconical appearance of the Red Rectangle is preserved on scales from 50 mas to $1^{\prime}$ and from the visible (red) to at least $10 \mu \mathrm{m}$, implying that large grains of at least several microns in size dominate scattering. The new images supplement previous 76 mas resolution speckle reconstructions at shorter wavelengths of 0.6-0.8 $\mu \mathrm{m}$ (Osterbart et al. 1997) and 0.7-2.2 $\mu \mathrm{m}$ (Men'shchikov et al. 1998), allowing a more detailed analysis of the famous bipolar nebula. The intensity distribution of the images is inconsistent with a flat disk geometry frequently used to model the bipolar nebulae. Instead, a geometrically thick torus-like density distribution with bipolar conical cavities is preferred. The extent of the bright lobes indicates that the dense torus has a diameter of $\gtrsim 100 \mathrm{AU}$, for an assumed distance of $330 \mathrm{pc}$. This torus may be the outer reaches of a flared thick disk tapering inwards to the central star, however such a density enhancement on the midplane is not strictly required to explain the narrow dark lane obscuring the central stars.
\end{abstract}

Key words. techniques: image processing - circumstellar matter - stars: individual: Red Rectangle stars: mass-loss - stars: AGB and post-AGB - infrared: stars

\section{Introduction}

The Red Rectangle is a well-known bipolar reflection nebula (HD 44179, AFGL 915, IRAS 06176-1036) around an evolved close binary system. The object has been extensively studied for almost three decades (see, e.g., references in Waters et al. 1998; Men'shchikov et al. 1998, hereafter MBOW), although a reliable model of the central close binary and its dusty environment is yet to be determined. To reconstruct the physical properties of the circumbinary torus and bipolar outflow causing the famous

Send offprint requests to: P. G. Tuthill, e-mail: gekko@physics.usyd.edu.au
X-shaped appearance, we need multiwavelength observations of the Red Rectangle with highest possible resolutions. High-resolution images would provide the necessary observational basis for quantitative theoretical work aimed at deeper understanding of the formation and evolution of the Red Rectangle and similar objects. Such an unveiling of the central region of the bipolar nebula is key to discriminating which among the existing qualitative models of the object are most plausible.

The first high-resolution near-infrared study used deconvolved adaptive optics images of the $3.6 \mathrm{~m}$ CFHT (Roddier et al. 1995), revealing the X-shaped spikes to persist on subarcsecond scales and finding two bright lobes 
Table 1. Keck observations on December 18, 1997. Listed are central wavelengths $\lambda$ of the filters, their $F W H M$ bandwidths $\Delta \lambda$ and symbols used, numbers of pixels, numbers of object frames, exposure times per frame, number of reference frames, seeing, and resolutions of the reconstructed images.

\begin{tabular}{|c|c|c|c|c|c|c|c|c|}
\hline $\begin{array}{c}\lambda \\
(\mu \mathrm{m})\end{array}$ & $\begin{array}{c}\Delta \lambda \\
(\mu \mathrm{m})\end{array}$ & Symbol & $\begin{array}{c}\text { Number of } \\
\text { pixels }\end{array}$ & $\begin{array}{l}\text { Number of } \\
\text { frames }\end{array}$ & $\begin{array}{l}\text { Exposure } \\
\quad(\mathrm{ms})\end{array}$ & $\begin{array}{l}\text { Number of } \\
\star \text { frames }\end{array}$ & $\begin{array}{l}\text { Seeing } \\
\left({ }^{\prime \prime}\right)\end{array}$ & $\begin{array}{l}\text { Resolution } \\
\text { (mas) }\end{array}$ \\
\hline 2.12 & 0.02 & $K_{\mathrm{H}_{2}}$ & 256 & 194 & 149 & 197 & 1. & 44 \\
\hline 2.16 & 0.02 & $K_{\mathrm{Br}_{\gamma}}$ & 256 & 194 & 149 & 134 & 1. & 45 \\
\hline 2.26 & 0.05 & $K_{\mathrm{c}}$ & 256 & 100 & 149 & 193 & 0.8 & 47 \\
\hline 3.08 & 0.10 & $L_{\mathrm{c}}$ & 184 & 767 & 87 & 576 & 0.7 & 64 \\
\hline 3.31 & 0.06 & $L_{\mathrm{PAH}}$ & 184 & 498 & 87 & 596 & 0.8 & 68 \\
\hline
\end{tabular}

separated by a dark lane. A more recent series of optical and near-infrared $(0.6-2.2 \mu \mathrm{m})$ speckle images with 62-76 mas resolutions obtained with the $2.2 \mathrm{~m} \mathrm{ESO/MPG}$ telescope (Osterbart et al. 1997, hereafter OLW) and the $6 \mathrm{~m}$ SAO telescope in Russia (MBOW) unveiled more details of the subarcsecond environment of the Red Rectangle, across a wider wavelength range. A compact, highly symmetric bipolar nebula with two bright scattering lobes, X-shaped spikes, and no direct light from the completely obscured close binary implied an optically thick, toroidal distribution of the circumbinary material. Both the speckle images and the spectral energy distribution (SED) of the Red Rectangle were studied in MBOW using self-consistent two-dimensional radiative transfer modeling, to derive geometry and physical parameters of the object. The authors concluded that the close binary with a total luminosity of $L_{\star} \approx 3000 L_{\odot}$ (for an assumed distance of $D=330 \mathrm{pc}$ ) is located inside a compact $(R \sim 30 \mathrm{AU})$, massive $\left(M=0.25 M_{\odot}\right)$, opaque $\left(A_{V} \approx 30\right)$ dusty torus of the Red Rectangle composed of predominantly very large (millimeter-sized) grains. The bipolar, cone-shaped outflow cavities, with a full opening angle of $\omega \approx 70^{\circ}$, reproduce the observed appearance of the nebula if the toroidal structure is observed almost edge-on $\left(\right.$ at $\theta_{\mathrm{v}} \approx 7^{\circ}$ ).

This paper improves the previous work by achieving highest ever resolutions of $46-68$ mas at longer wavelengths $(2-3.3 \mu \mathrm{m})$. In Sect. 2 we describe the observations. In Sect. 3 we present diffraction-limited speckle images of the Red Rectangle and in Sect. 4 we discuss and interpret the new images on the basis of the model published by MBOW. In Sect. 5 we summarize results of this study.

\section{Observations}

The observations were obtained at the $10 \mathrm{~m}$ Keck telescope on December 18/19, 1997 (JD 2450801). The speckle interferograms were recorded with the Keck facility nearinfrared camera (NIRC), using external magnifying optics (image converter, Matthews et al. 1996). The plate scale was 20.57 mas pixel $^{-1}$, sufficient to Nyquist sample the data collected in the $K$ band and at longer wavelengths. The field of view for the $256 \times 256 \mathrm{InSb}$ array was 5 ". 3 . For observations beyond $3 \mu \mathrm{m}$, the increasing thermal background made it desirable to shorten exposure times, which was accomplished by reading only $184 \times 184\left(3^{\prime \prime} 8\right)$ subframes. Data sets were interleaved between the source and a nearby point-source reference star, HD 41971, which was used to calibrate the telescope-atmosphere transfer function. Further details on the readout methods employed can be found in Tuthill et al. (2000). The filters were selected from NIRC's standard complement of interference filters which offered a range of bandwidths (1-20\%) covering the $1-3.5 \mu \mathrm{m}$ region. More details on the filters, their full widths at half-maximum $(F W H M)$, numbers of pixels and speckle frames, frame integration time, seeing, and resolutions can be found in Table 1 .

\section{Reconstructed images}

Diffraction-limited images of the Red Rectangle were reconstructed from the speckle interferograms using the bispectrum speckle interferometry method (Weigelt 1977; Lohmann et al. 1983; Weigelt 1991). The power spectrum of the object was determined with the speckle interferometry method (Labeyrie 1970).

The reconstructed speckle images of the Red Rectangle are presented in Fig. 1. At all wavelengths we observed continuum radiation from the Red Rectangle with the exception of the filter centered at $3.31 \mu \mathrm{m}$, where around $20-30 \%$ of the emission received is from strong line emission of polycyclic aromatic hydrocarbons (PAHs) as can be seen from spectra obtained with a $1^{\prime \prime} .2$ aperture pointing at the center of the nebula (Kerr et al. 1999). We presume that essentially all of the radiation in the $K_{\mathrm{Br}_{\gamma}}$ filter is continuum, although there may be very little of $\mathrm{Br}_{\gamma}$ emission from the Red Rectangle observed in a $17^{\prime \prime}$ aperture, as is seen from spectrophotometry by Russell et al. (1977). We do not separately display the $K_{\mathrm{H}_{2}}, K_{\mathrm{Br}_{\gamma}}$, and $K_{\mathrm{c}}$ images in Fig. 1, because they are practically indistinguishable from each other. Instead, they have been co-added into a single $K_{\mathrm{S}}$ image to improve the signal-to-noise ratio.

High-resolution color images have proven to be very useful for detailed interpretation of complex bipolar structures (see, e.g., Men'shchikov et al. 2001). Therefore, in Fig. 1, we also present the $H-K_{\mathrm{S}}, H-L_{\mathrm{c}}$, and $K_{\mathrm{S}}-L_{\mathrm{c}}$ color images of the Red Rectangle derived from 

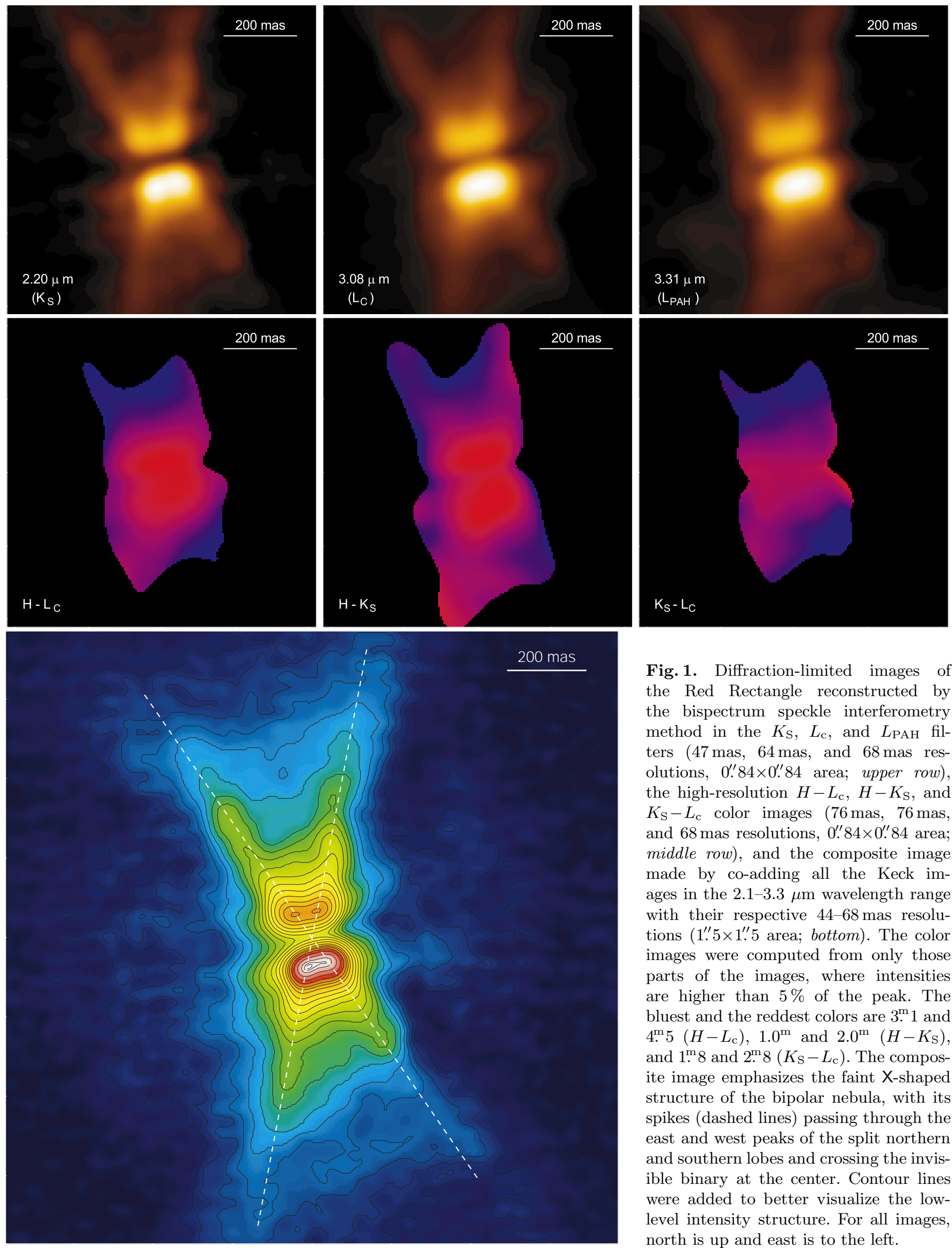

Fig. 1. Diffraction-limited images of the Red Rectangle reconstructed by the bispectrum speckle interferometry method in the $K_{\mathrm{S}}, L_{\mathrm{c}}$, and $L_{\mathrm{PAH}}$ filters (47 mas, 64 mas, and 68 mas resolutions, $0 . .84 \times 00^{\prime \prime} 84$ area; upper row), the high-resolution $H-L_{\mathrm{c}}, H-K_{\mathrm{S}}$, and $K_{\mathrm{S}}-L_{\mathrm{c}}$ color images $(76 \mathrm{mas}, 76 \mathrm{mas}$, and 68 mas resolutions, $00^{\prime \prime} 84 \times 00^{\prime \prime} 84$ area; middle row), and the composite image made by co-adding all the Keck images in the $2.1-3.3 \mu \mathrm{m}$ wavelength range with their respective 44-68 mas resolutions $\left(1^{\prime \prime} .5 \times 1^{\prime \prime} .5\right.$ area; bottom). The color images were computed from only those parts of the images, where intensities are higher than $5 \%$ of the peak. The bluest and the reddest colors are $3 .{ }^{\mathrm{m}} 1$ and $4^{\mathrm{m}} 5\left(H-L_{\mathrm{c}}\right), 1.0^{\mathrm{m}}$ and $2.0^{\mathrm{m}}\left(H-K_{\mathrm{S}}\right)$, and 1.8 and $2.8\left(K_{\mathrm{S}}-L_{\mathrm{c}}\right)$. The composite image emphasizes the faint $\mathrm{X}$-shaped structure of the bipolar nebula, with its spikes (dashed lines) passing through the east and west peaks of the split northern and southern lobes and crossing the invisible binary at the center. Contour lines were added to better visualize the lowlevel intensity structure. For all images, north is up and east is to the left. 
our multiwavelength speckle data obtained with the $10 \mathrm{~m}$ Keck telescope and the $6 \mathrm{~m}$ SAO telescope (MBOW). The full interpretation of such color maps requires detailed modeling of the interplay between opacity, scattering and thermal effects, however in this case it is likely that bluer areas are more scattering dominated, whereas red areas represent thermal emission from hot dust. The images in the $L_{\mathrm{PAH}}$ and $L_{\mathrm{c}}$ filters are quite similar (Fig. 1), although some differences were noted in the more extended emission. As these were in areas of relatively low signal, we have chosen not to include the $L_{\mathrm{PAH}}-L_{\mathrm{c}}$ color image and await independent confirmation of the changes.

The images in Fig. 1 confirm the earlier images with a slightly lower resolution presented and modeled by MBOW. However, our new images reveal details of the intensity distribution of the closest environment of the circumbinary torus with higher resolution and at longer wavelengths. To enable a more quantitative analysis of the images, we present in Fig. 2 intensity cuts through the lobes along the symmetry axis of the bipolar nebula $\left(\mathrm{PA}=12^{\circ}\right)$ and in a perpendicular direction.

\section{Discussion}

\subsection{Morphology}

Two well-resolved bright lobes above and below the inclined torus of the Red Rectangle are seen in Fig. 1, along with the $\mathrm{X}$-shaped spikes originating deep inside the biconical outflow cavities. The presence of the torus is imprinted on the images in the central dark band produced by the optically thick equatorial regions viewed almost edge-on, and in the lateral extent of the structure delimited by the overall size of the dense dust. The spikes are bright enough to contribute to the intensity distribution of the bright lobes (outflow cavities), making them appear broadened and even showing an east-west double peak in the Fig. 1 images with the highest resolution (see also Fig. 2). Such splitting of the lobes has never been seen before. The physical cause of the $\mathbf{X}$-shaped spikes is most likely a combination of the limb brightening and scattering by dust grains in the dense boundaries of the biconical outflow cavities (MBOW). Polarization maps by Perkins et al. (1981) suggested that the spikes are produced by dust scattering at dense surfaces of the bicone. Unidentified optical emission bands, extended red emission, and $3.3 \mu \mathrm{m}$ PAH emission line (of Type I) also originate close to the biconical surfaces along the $X$ spikes (Kerr et al. 1999).

The spikes are clearly seen in all images, traced along the surface of the biconical outflow cavities to within the distances of 0 .' 4 from the center of the nebula. Their appearance is very similar to that of the $H$ - and $K$-band images presented by MBOW, where the spikes are visible up to $0^{\prime \prime} 5$. It is clear that the length of the spikes depends on the density distribution, dust properties, as well as on the sensitivity and resolution of observations. To further increase the signal-to-noise ratio and better see the faint $X$ structure, we have co-added all the Keck images at their respective resolutions. The composite near-IR image in Fig. 1 emphasizes the low-level intensity distribution and enables us to trace the spikes to within 0 .' 8 from the central binary. The spikes can be approximated by straight lines passing through the east and west peaks of the split northern and southern lobes and intersecting at the position of the central binary. From the image, we can determine the opening angle of the conical outflow cavities $\omega \approx 50^{\circ}$ and the position angle of the symmetry axis on the sky $\mathrm{PA} \approx 12^{\circ}$. The values differ somewhat from the $\omega$ of $40^{\circ}$ and $60^{\circ}$ and the $\mathrm{PA}$ of $14^{\circ}$ and $15^{\circ}$ of the $1^{\prime \prime}$ and $4^{\prime \prime}$ areas of the images presented by MBOW, as well as from the adopted $\omega=70^{\circ}$ of their model. The fact that our composite image in Fig. 1 traces an intermediate area of $1.5^{\prime \prime}$ suggests that the $\mathrm{X}$ spikes have indeed a noticeable curvature, with the cavity widening on larger scales.

The X-shaped spikes of the Red Rectangle are qualitatively very similar to the X-shaped "searchlight beams" of the proto-planetary nebula CRL 2688 (the Egg Nebula, Sahai et al. 1998), the bipolar outflow cavity of the latter being almost twice narrower $\left(\omega \approx 30^{\circ}\right)$. Both these objects are similar to the evolved carbon star IRC +10 216 (Men'shchikov et al. 2001), although in the latter such strong X-shaped spikes have not been observed, possibly because of different dust properties and viewing angle. A unique property of the Red Rectangle is that even the $L_{\mathrm{c}}$ and $L_{\mathrm{PAH}}$ images show almost the same intensity distribution as the images at shorter wavelengths (OLW; MBOW and Fig. 1). Moreover, the biconical shape is preserved across a wide range of wavelengths $(0.6-10 \mu \mathrm{m})$ and spatial scales (0.001-1') (cf. Cohen et al. 1975; Perkins et al. 1981; Waters et al. 1998; Men'shchikov et al. 1998; Van Winckel 2001). This fact provides a strong observational constraint on the density structure and dust properties of the circumbinary environment of the Red Rectangle.

The invariant biconical shape of the nebula at such different scales and even at the mid-infrared wavelengths suggests that the shape is produced by the same mechanism, namely, by scattering. Mid-IR images in which the bipolar outflow can be traced to more than $5 \times 10^{3} \mathrm{AU}$ from the central stars rule out thermal emission from dust: grains are too cold at these distances. Both at the optical and near-IR wavelengths, the biconical shape is caused by the radiation scattered by dust grains in the bipolar outflow. The dust grains must be very large compared to the normal (small) interstellar grains since the latter have negligible albedo in the mid-infrared. Dominant grains must have sizes of at least several microns in order to efficiently scatter the mid-IR radiation and to be able to explain our images. Modeling of the entire SED of the Red Rectangle points to the presence of even much larger, millimetersized grains in its dense environment (MBOW).

The bipolar appearance of the Red Rectangle implies that the density in the outflow cones is much lower than that of the dense torus in the innermost subarcsecond region (MBOW). The inner dense torus is optically thick $(\tau \approx 30)$ and the observed appearance of the Red Rectangle requires the outflow cavities to be optically thin 

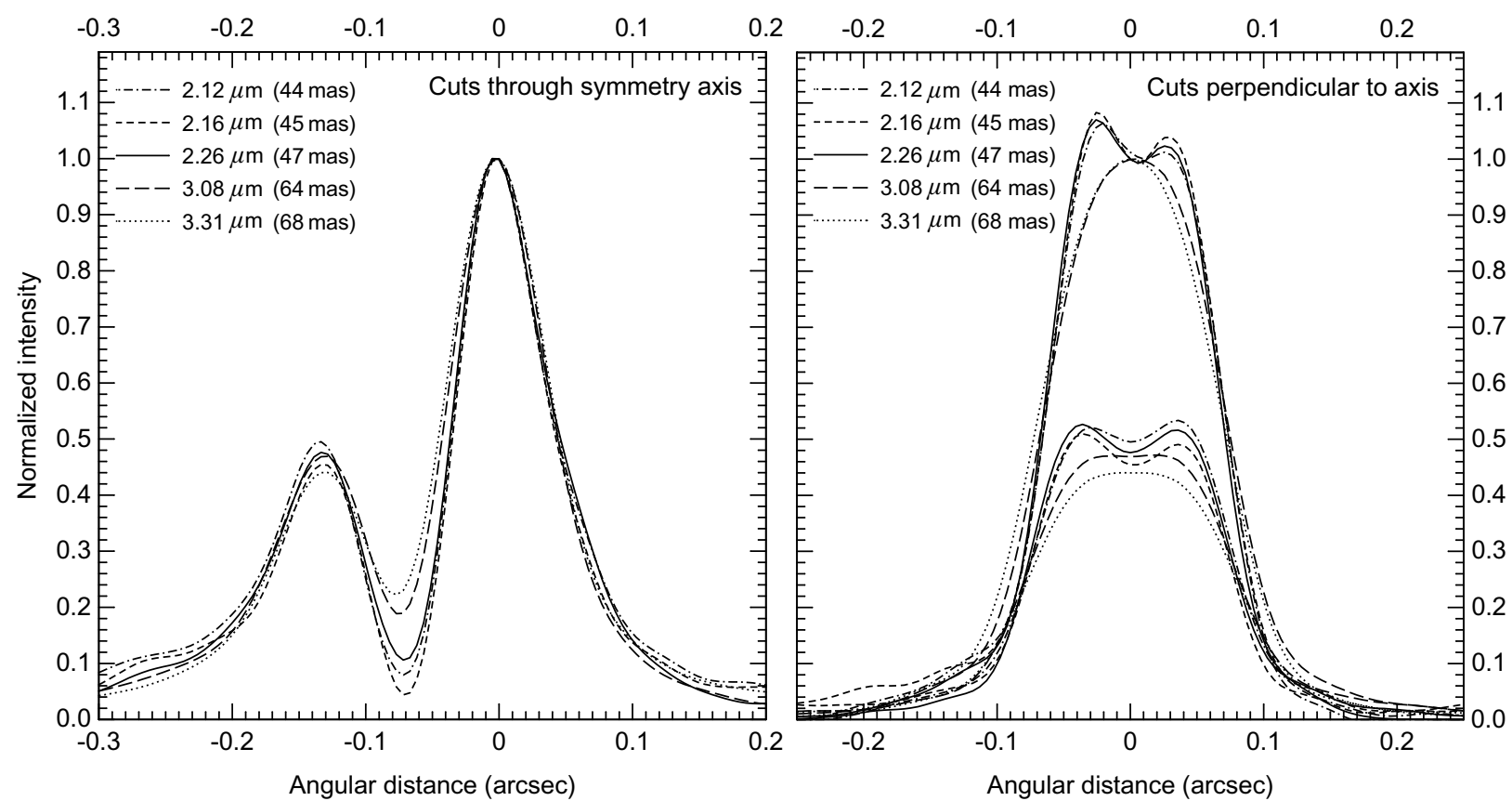

Fig. 2. Normalized intensity profiles derived from our speckle images of the Red Rectangle shown along the symmetry axis $\left(\mathrm{PA}=12^{\circ}\right.$, left panel) and across the lobes in the perpendicular direction $\left(\mathrm{PA}=102^{\circ}\right.$, right panel). For the cuts along the axis, the centerline of the nebula was chosen. The two sets of perpendicular cuts shown pass through the brightest parts of the northern (dimmer) and southern (brighter) lobes.

$(\tau \lesssim 0.1)$. On the other hand, the outer parts of the circumbinary material (at angular distances $\theta \gtrsim 0.5$ ) are optically thin in all directions (MBOW). This suggests that the strong biconical shape visible in the outer parts of the Red Rectangle (beyond the field of images presented here) is produced by dust scattering in the outflow regions, which are much denser there than the rest of the torus at the same distance from the center. This conclusion implicitly assumes that the dust properties in the outflow cones and in the rest of the toroidal envelope are the same.

\subsection{Disk, torus, or envelope?}

Our near-IR images of the Red Rectangle (Fig. 1) clearly show the bright southern and fainter northern lobes associated with a geometrically and optically thick disk or torus inclined toward us by $\sim 10^{\circ}$ (MBOW). As is becoming more apparent with higher resolutions, the bright lobes are divided by a very dark narrow lane. The images exhibit a compact, highly symmetric brightness distribution, very similar in shape and extent for all five wavelengths. In agreement with the results of previous speckle imaging $(\mathrm{MBOW})$, the relative peak intensities of the fainter (northern) lobe in the new images are in the range of $45-50 \%$ of the brighter (southern) lobe, almost independent of the wavelength (Fig. 2). Such an appearance suggests a strong concentration of circumstellar matter toward the central binary in an axially-symmetric density distribution (MBOW) and a high gray optical depth due to large dust particles (Sect. 4.3).
We would like to emphasize that, despite the narrowness of the dark lane, the circumbinary material we see in the images is definitely not confined to a flat disk. The appearance of the optically thick structures with bipolar outflow cavities is often misleading (cf. Men'shchikov \& Henning 2000; Men'shchikov et al. 2001). The narrow dark lane is a result of the radiation transfer effects, with optical depths along different lines of sight greatly varying in such an axisymmetric density distribution (Fig. 8 in MBOW). The narrow dark lane appears quite naturally in toroidal bipolar envelopes even when the torus density is independent of the polar angle (i.e. extra density along the midplane is not required, MBOW). This is also demonstrated by our $H-K_{\mathrm{S}}, H-L_{\mathrm{c}}$, and $K_{\mathrm{S}}-L_{\mathrm{c}}$ color images (Fig. 1), which show a red plateau of size approximately $200 \times 200$ mas located over the dark lane and bright lobes. We identify this plateau as mostly thermal emission from dust in a thick disk or torus.

A thin flat disk observed edge-on would appear as an ellipsoidal structure highly elongated at $\mathrm{PA}=102^{\circ}$ (by a factor of $\sim 5$ ) perpendicular to the symmetry axis of the nebula (MBOW), with a narrow and long dark lane. For an illustration of this statement, we refer the reader to the $L$-band model images of a circumstellar disk in Suttner \& Yorke (2001). The expected appearance of a thin disk sharply contrasts the observed bipolar brightness distribution extended along the symmetry axis. This rules out the flat disk as a model for the interpretation of the observed properties of the Red Rectangle and other similar bipolar nebulae. Plausible geometries of the distribution of dusty material may include either a strongly flaring, 

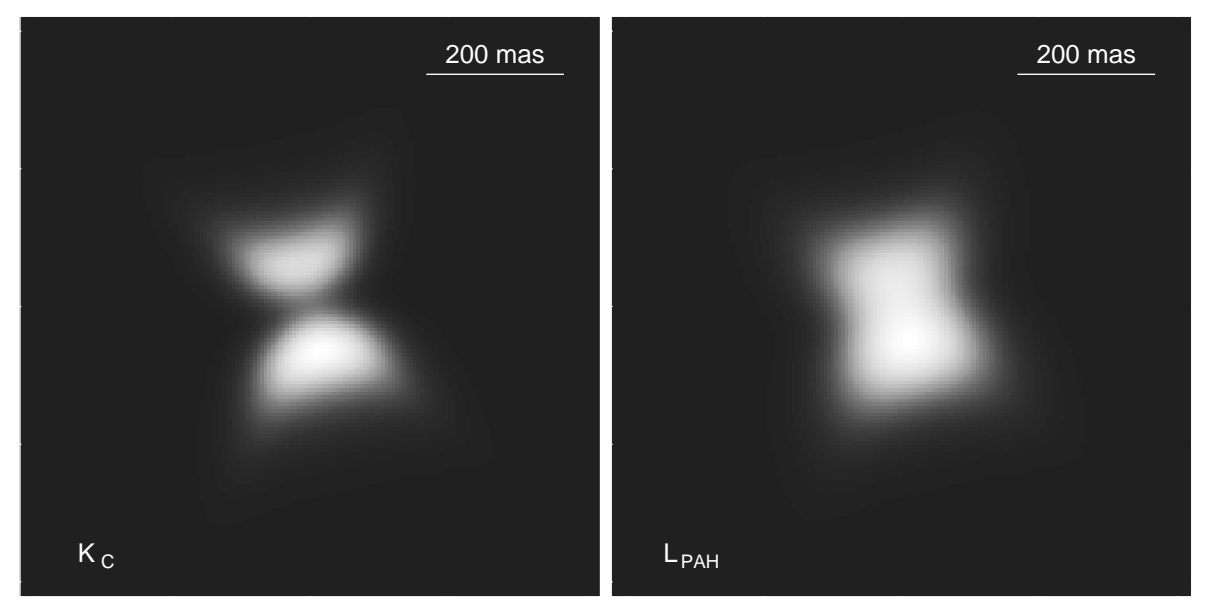

Fig. 3. Predicted images of the Red Rectangle model (MBOW) in $K_{\mathrm{c}}$ (left panel) and $L_{\mathrm{PAH}}$ (right panel) bands, convolved to the 47 mas and 68 mas resolutions of our Keck images. Whereas the $K_{\mathrm{c}}$ image is very similar to the observed one, the $L_{\mathrm{PAH}}$ image shows no dark lane at all, indicating that the optical depth of the model torus is insufficient to explain the new data.

geometrically very thick disk or a toroidal structure with biconical outflow cavities (MBOW).

\subsection{Dust grains}

The peak brightness ratio of the two bipolar lobes is almost the same across the wide wavelength range of at least $0.6-3.3 \mu \mathrm{m}$ (see Fig. 7 in MBOW and Fig. 2 in this paper). This suggests that the optical depth is gray in this wavelength range, which means that dominant grains are larger than $1 \mu \mathrm{m}$ in radius. If we recall, however, that the biconical shape of the nebula is visible even in the midIR $N$ band (at $10 \mu \mathrm{m}$, Waters et al. 1998), we can conclude that there are sufficient grains with radii of at least several microns. Modeling of the spectral energy distribution together with optical and near-IR images of the Red Rectangle has indicated that millimeter-sized grains dominate the gray circumstellar extinction at all wavelengths up to $\sim 1 \mathrm{~cm}(\mathrm{MBOW})$. As it is difficult to imagine that there are no small grains at all, most likely a wide distribution of grain sizes exists in the Red Rectangle, with a size distribution much flatter than that in the interstellar medium. Largest grains with gray extinction naturally dominate scattering in such a population of particles.

The dark lane between the lobes has a similar width in all the images, although the highest-resolution $K_{\mathrm{H}_{2}}$ and $K_{\mathrm{Br}_{\gamma}}$ images clearly resolve it deeper (cf. Fig. 2, left). Even the latter show no signs of either the direct light from the central binary, or an increasing contribution of hot emission $\left(T_{\mathrm{d}} \sim 1000 \mathrm{~K}\right)$ from the inner dust boundary of the torus. This is another indication of high optical depths due to the presence of large grains. If the dark lane at short (visible) wavelengths was produced by typical interstellar grains, its $L_{\mathrm{c}}$ and $L_{\mathrm{PAH}}$ band optical depths would be significantly lower. In that case, we would see deeper through the torus and the emission of the hot grains would show up in the images, producing a single compact peak. There are almost no changes in the images over all the nearIR wavelengths except for those caused by just somewhat different resolutions (Fig. 2, left). In fact, if we achieved a 45 mas resolution in the $L_{\mathrm{c}}$ and $L_{\mathrm{PAH}}$ bands, the corresponding minimum intensities in the dark lane would be very similar to those of the other bands. Such an invariance of the intensity profiles indicates that very large grains dominate the scattered and emitted radiation in the very dense circumbinary torus of the Red Rectangle (cf. MBOW).

To illustrate these statements, in Fig. 3 we show the $K_{\mathrm{c}}$ and newly-computed $L_{\mathrm{PAH}}$ band images of the radiative transfer model of the Red Rectangle presented by MBOW. The model was constrained by the SED and highresolution optical and near-IR images up to the $K$ band. To check whether the model is also consistent with the new constraints, we calculated the images at the wavelengths of our Keck images and convolved them to the corresponding resolutions. An inspection of Fig. 3 shows that the model explains the new highest-resolution images up to the $K_{\mathrm{c}}$ band quite well, whereas at longer wavelengths it predicts a single intensity peak extended along the symmetry axis. This means that the optical depth of the model is insufficient to explain the new Keck images in $L_{\mathrm{c}}$ and $L_{\mathrm{PAH}}$ filters. In the model, there is too much emission coming out from the hot dust grains close to the inner boundary of the torus. On the basis of their model, MBOW predicted that the direct light from the binary could be visible in the near IR with a significantly better resolution. However the new observational constraint requiring significantly higher optical depths of the torus invalidates this specific prediction and explains why the star was not seen there. Still higher sensitivity will be required to detect the direct stellar light. The model has been modified to include the new constraints (Men'shchikov et al. 2002, in preparation).

\section{Conclusions}

We presented new diffraction-limited images of the Red Rectangle in several filters in the wavelength 
range $2.1-3.3 \mu \mathrm{m}$ with the angular resolution as high as 44-68 mas. These images imply a well-resolved inclined, optically and geometrically thick flared disk or torus of $\sim 200$ mas diameter, with bright lobes above and below its midplane, and $\mathrm{X}$-shaped spikes along the surfaces of the conical outflow cavities. Assuming a distance of $330 \mathrm{pc}$ to the Red Rectangle, the dense torus has an outer diameter of $\gtrsim 100 \mathrm{AU}$.

Confirming results of modeling by MBOW, the new images show intensity distributions inconsistent with a flat disk geometry frequently used to visualize the bipolar nebulae. Geometrically thick density distributions with strongly flaring disks or a torus with bipolar conical cavities are best suited for reproducing the observed images. A remarkable feature of the Red Rectangle bipolar nebula is its self-similar appearance on scales from 50 mas to $1^{\prime}$ and from the red (visible) to at least $10 \mu \mathrm{m}$. It implies that most of the dust mass in the dense torus is in very large grains of at least several microns in size and that the outflow regions are denser than the toroidal envelope surrounding it on scales of more than an arcsecond. Taken together with existing high-resolution speckle images at shorter wavelengths, the new images allow a more accurate interpretation of the shape of the bipolar nebula. Self-consistent two-dimensional radiative transfer modeling of the Red Rectangle will be presented in a subsequent paper (Men'shchikov et al. 2002, in preparation).

Acknowledgements. The data presented herein were obtained at the W.M. Keck Observatory, which is operated as a scientific partnership among the California Institute of Technology, the University of California and the National Aeronautics and Space Administration. The Observatory was made possible by the generous financial support of the W.M. Keck Foundation. We thank the referee, Dr. R. R. Howell, for comments that helped improve clarity of the paper.

\section{References}

Cohen, M., Anderson, C. M., Cowley, A., et al. 1975, ApJ, 196, 179

Kerr, T. H., Hurst, M. E., Miles, J. R., \& Sarre, P. J. 1999, MNRAS, 303, 446

Labeyrie, A. 1970, A\&A, 6, 85

Lohmann, A. W., Weigelt, G., \& Wirnitzer, B. 1983, Appl. Opt., 22, 4028

Matthews, K., Ghez, A. M., Weinberger, A. J., \& Neugebauer, G. 1996, PASP, 108, 615

Men'shchikov, A. B., Balega, Y. Y., Blöcker, T., Osterbart, R., \& Weigelt, G. 2001, A\&A, 368, 497

Men'shchikov, A. B., Balega, Y. Y., Osterbart, R., \& Weigelt, G. 1998, NewAst, 3, 601 (MBOW)

Men'shchikov, A. B., \& Henning, T. 2000, in Darwin and Astronomy - The Infrared Space Interferometer, ed. B. Schürmann, Proc. Conf. "Darwin and Astronomy", Stockholm, 17-19 Nov. 1999 (ESA SP-451, May 2000) (Noordwijk: ESA Publications Division), 125

Osterbart, R., Langer, N., \& Weigelt, G. 1997, A\&A, 325, 609 (OLW)

Perkins, H. G., Scarrott, S. M., Murdin, P., \& Bingham, R. G. 1981, MNRAS, 196, 635

Roddier, F., Roddier, C., Graves, J. E., \& Northcott, M. J. 1995, ApJ, 443, 249

Russell, R. W., Soifer, B. T., \& Merrill, K. M. 1977, ApJ, 213, 66

Sahai, R., Trauger, J. T., Watson, A. M., et al. 1998, ApJ, 493, 301

Suttner, G., \& Yorke, H. W. 2001, ApJ, 551, 461

Tuthill, P. G., Monnier, J. D., Danchi, W. C., Wishnow, E. H., \& Haniff, C. A. 2000, PASP, 112, 555

Van Winckel, H. 2001, Ap\&SS, 275, 159

Waters, L. B. F. M., Waelkens, C., van Winckel, H., et al. 1998, Nature, 391, 868

Weigelt, G. 1991, in Progress in Optics, ed. E. Wolf, vol. XXIX (Amsterdam: Elsevier Science Publishers), 295

Weigelt, G. P. 1977, Opt. Commun., 21, 55 